\title{
Radiopharmaceuticals for cardiac imaging: Current status and future trends
}

\author{
Raymond Taillefer, MD, FRCP, ABNM, a and Francois Harel, MD, PhD ${ }^{b}$ \\ a Département d’imagerie médicale, Hôpital du Haut-Richelieu du CISSS Montérégie-Centre, \\ Saint-Jean-sur-Richelieu, QC, Canada \\ b Département d'imagerie médicale, Institut de Cardiologie de Montréal, Montreal, QC, Canada
}

Received Dec 10, 2017; accepted Dec 11, 2017

doi: $10.1007 / \mathrm{s} 12350-018-1194-y$

\section{See related article, pp. 1204-1236}

Although nuclear cardiology is generally considered as a clinical specialty since approximately four decades, the initial application of radioisotopes to the study of blood circulation in humans (pulmonary circulation time) was reported almost a century ago in 1927. Blumgart and Weiss ${ }^{1}$ used radon gas dissolved in saline solution as the radiotracer and a modified Wilson cloud chamber as the radiation detector. Two decades later, in 1949, Prinzmetal et al. ${ }^{2}$ used sodium iodide probes and radiolabeled albumin to measure cardiac output and blood volumes. These important initial studies provided the roots of nuclear cardiology which extended its applications not only to heart function and blood circulation but now to the non-invasive imaging of myocardial perfusion, infarct, myocardial viability, cardiac inflammation, and cardiac metabolism with the use of various radiotracers.

\section{MYOCARDIAL PERFUSION IMAGING}

Myocardial perfusion imaging (MPI) represented and still represents the vast majority of nuclear cardiologic procedures performed every year in clinical laboratories throughout the world. Myocardial perfusion scintigraphy has significantly evolved since its introduction more than four decades ago. ${ }^{3}$ Three major

Reprint requests: Raymond Taillefer, MD, FRCP, ABNM, Département d'imagerie médicale, Hôpital du Haut-Richelieu du CISSS Montérégie-Centre, Saint-Jean-sur-Richelieu, QC, Canada; rtaillefer@hotmail.com

J Nucl Cardiol 2018;25:1242-6.

$1071-3581 / \$ 34.00$

Copyright (c) 2018 American Society of Nuclear Cardiology. factors have more specifically contributed to this evolution: (1) technical improvements in SPECT scintigraphic data acquisition and analysis, (2) introduction of new technetium-99m-labeled MPI radiopharmaceuticals with different properties than thallium-201, and (3) more recently, availability of PET radiotracers and PET dedicated cameras. In the last four decades, several studies have been performed on various types of radiotracers but we can ask the question: why do we still need new radiotracers for MPI? After all, four SPECT radiotracers have already been approved by the Food and drugs administration (FDA) for clinical use: ${ }^{201}$ Thallium, ${ }^{99 m}$ Tc-Sestamibi, ${ }^{99 \mathrm{~m}}$ Tc-Tetrofosmin, and ${ }^{99 \mathrm{~m}} \mathrm{Tc}-\mathrm{Teboroxime}$, and two PET agents (Rubidium-82 and N-13 Ammonia) as well. Table 1 summarizes the most important characteristics of an «ideal»SPECT or PET radiotracer for MPI. Although the currently available radiotracers show many of the characteristics of the «ideal » radiotracer, none of them have all the desired ones. All of the current FDA-approved SPECT radiotracers show, at variable degree, the « roll-off» phenomenon at high flow rates which can limit the detection of ischemia in some patients with multivessel disease or mild-to-moderate CAD, especially at high flow rates, as those seen with pharmacological intervention. ${ }^{4,5}$

PET radiotracers are increasingly used for MPI, given the broader clinical access to PET cameras. ${ }^{6} \mathrm{~N}$ 13-Ammonia is a valuable radiotracer for assessing either relative myocardial uptake or measuring absolute myocardial blood flow (with dynamic acquisition and application of multiple-compartment kinetic models). However, the use of N-13-Ammonia requires an on-site cyclotron (because of its physical half-life of $10 \mathrm{~min}$ ute), thus limiting its use to a very restricted number of sites. Rubidium-82, another PET MPI radiotracer, does not require on-site cyclotron as it is produced with a Strontium-82/Rubidium-82 generator. ${ }^{7}$ Furthermore, 
Table 1. Criteria for the "ideal " SPECT and PET myocardial perfusion imaging radiotracer

(A) Physiological characteristics

1. High myocardial uptake with no or minimal myocardial redistribution, especially during the data acquisition time period

2. Linear relationship between radiotracer myocardial uptake and coronary blood flow (up to $5 \mathrm{~mL} / \mathrm{min} / \mathrm{g}$, which is the maximal level seen with pharmacological vasodilation). This requires a high first-pass myocardial extraction and subsequent very rapid blood clearance

3. A high target-to-background ratio with low uptake in the adjacent organs (lungs, liver, stomach, and bowels. This high ratio should be maintained throughout the entire imaging protocol, especially if a same day rest-stress or stress-rest protocol is to be performed

4. Quantification of absolute myocardial blood flow, enabling identification of diffuse, multivessel, or balanced CAD. Perhaps more importantly, absolute myocardial blood flow and coronary flow reserve identify patient with suboptimal response to pharmacological stress and provides incremental prognosis information over perfusion alone

5. The effect of blood flow on myocardial transport of the radiotracer must be predominant to the effect of metabolic cellular alterations

(B) Physical characteristics

1. Appropriate radiation dosimetry for a complete rest-stress evaluation

2. Appropriate safety profile, especially considering repeated studies

3. A physical half-life that allows for enough time for adequate image acquisition (and repeated acquisition, if necessary) with both treadmill stress test and pharmacological intervention

4. Constant availability of the radiotracers at relatively low cost

5. Easy labeling procedures and stable labeling of the reconstituted radiotracer

6. For SPECT radiotracers

(a) Labeling to ${ }^{99 m}$ Technetium with favorable physical characteristics, providing high photon flux and optimal counting statistics

7. For PET radiotracers

(a) Shorter positron range that will provide better image quality

(b) Availability as unit dose, preferably labeled with Fluorine-18 because it does not require an on-site cyclotron or expensive generator

Rubidium-82 possesses the unique advantage of having a very short half-life of 75 second, allowing for a complete rest-stress MPI study within 30 minute. Although qualitative assessment of relative Rubidium82 perfusion defects shows a good correlation with those obtained with radiolabeled microspheres or O-15 water, and that the diagnostic accuracy of Rubidium-82 imaging in clinical detection of CAD is high, net myocardial uptake of this radiotracer plateaus at the hyperemic flows often achieved with pharmacological stress. Furthermore, its very long positron range decreases the spatial resolution and Rubidium-82 has a lower myocardial extraction than N-13-Ammonia. Therefore, it is not surprising to see a quest for the ideal or near optimal MPI PET radiotracer. One very promising agent, ${ }^{18} \mathrm{~F}$ Flurpiridaz, an analog of mitochondrial complex I inhibitors, is currently under clinical investigation (Phases III). This radiotracer possesses many of the characteristics of the «ideal » MPI radiotracer although clinical data on its diagnostic accuracy are still needed before we can conclude on its future in clinical practice as the best available MPI radiotracer. ${ }^{9-11}$ Because of the longer half-life of ${ }^{18} \mathrm{~F},{ }^{18} \mathrm{~F}$-Flurpiridaz does not require an on-site cyclotron. Results of the ${ }^{18}$ F-Flurpiridaz phase III trials would be of high importance to determine its future clinical roles.

In the mean time, it could be interesting to see if it is possible to give a « second birth » to an older compound which has been approved by the FDA a few weeks after ${ }^{99 \mathrm{~m}} \mathrm{Tc}$-sestamibi has been approved in the early 1990s, ${ }^{99 \mathrm{~m}}$ Tc-Teboroxime, a member of the boronic acid adducts of technetium dioxime complexes (BATO) with a neutral charge and high lipophilicity. ${ }^{99 m}$ Tc-Teboroxime is a freely diffusible tracer with a very high extraction fraction over a wide range of coronary blood flow rates, higher than that of ${ }^{99 \mathrm{~m}} \mathrm{Tc}$ sestamibi, ${ }^{99 m}$ Tc-tetrofosmin, and ${ }^{201}$ Thallium. However, the myocardial half-life of ${ }^{99 \mathrm{~m}}$ Tc-teboroxime is approximately 9 minute, ${ }^{12,13}$ which gives a very narrow time window for optimal imaging (particularly with a liver clearance of 5-6 minute). This significant drawback limited the clinical use of this agent. However, 
with the introduction of new high-speed SPECT cameras with high counting sensitivity and new softwares that allow for fast image acquisition protocols and various types of image corrections to enhance the image resolution, it is possible to consider a « re-introduction » of ${ }^{99 \mathrm{~m}}$ Tc-Teboroxime into the clinical arena. This could be a perfect example to show the necessity to have a parallel development between the introduction of new radiotracers and the introduction of new types of imaging devices and/or new software and more sophisticated multicompartmental quantitative analyses.

\section{CARDIAC METABOLISM}

Different facets of cardiac metabolism have been studied with radiotracers. In the current issue of the Journal, Manabe et al. ${ }^{14}$ comprehensively reviewed the various radiotracers that are used to evaluate cardiac metabolism. Since the heart derives its energy from a variety of sources such as free fatty acids, glucose, lactate, and ketone bodies, it is possible to study cardiac metabolism using these sources. SPECT radiotracers include ${ }^{123}$ I-BMIPP (beta-methyl-p-iodophenylpentadecanoic acid) and ${ }^{123}$ I-IPPA (iodophenylpentadecanoic acid), whereas PET tracers include ${ }^{18} \mathrm{~F}-\mathrm{FDG}$ (fluorodeoxyglucose), ${ }^{11} \mathrm{C}$-Palmitate, ${ }^{18}$ F-FTHA (fluoro-6thia-heptadecanoic acid), and ${ }^{11} \mathrm{C}$-Acetate.

Although SPECT imaging of fatty acid myocardial metabolism with ${ }^{123}$ I-BMIPP and ${ }^{123}$ I-IPPA has gained in popularity in Japan, there are not still approved by the FDA, limiting their clinical applications. ${ }^{123}$ I-IPPA demonstrates a rapid accumulation in the heart and a clearance pattern that follows a biexponential function characteristic of ${ }^{11} \mathrm{C}$-Palmitate. The clearances rate correlates directly with $B$-oxidation. Unfortunately, the poor temporal resolution of SPECT systems did not take advantage of the rapid turnover of ${ }^{123}$ I-IPPA and quantification of myocardial fatty metabolism was not possible and image quality was reduced. ${ }^{123}$ I-BMIPP, a branch-chain analog of ${ }^{123}$ I-IPPA, ${ }^{15}$ shows increased radiotracer retention and improved image quality but is it not optimal. ${ }^{16}$ Here, again, the new SPECT technology with fast data acquisition and new image-qualityimproving methods and software could be advantageous with these « older» radiotracers. With technical improvements, fatty acid metabolic imaging could be more useful, especially in cardiac risk stratification and follow-up of treatment of patients with diabetes mellitus, obesity, insulin resistance, hypertension, left ventricular hypertrophy, non-ischemic dilated cardiomyopathy, and ischemia (detection of «ischemic memory »). ${ }^{17}$ In order to become an indispensible clinical tool in the diagnosis, risk stratification, and monitoring of therapy in many specific types of populations, radionuclide evaluation of the cardiac metabolism will need to move beyond viability assessment. This can be achieved only with large specific clinical trials where new technologies would be applied.

Non-invasive radionuclide metabolic imaging is a perfect example of the continued necessity for a parallel development in dedicated radiotracers, improved imaging devices, and application of more complex compartmental modeling which, ultimately, will permit more complete characterization of the metabolism of a given substrate to analyze. Dynamic data acquisitions with further increased counting statistics will be essential if optimal quantitative parameters are to be used in a clinical environment and have a real clinical impact in the day-to-day practice. Ideally, new radiotracers will be designed in order to provide new clinically oriented insights into the linkage between various substrate metabolism and cell growth, cell survival, and energy transfer.

\section{CARDIAC INNERVATION}

The heart is richly innervated by sympathetic and parasympathetic fibers. Neurohormonal processes control cardiac output, vascular tone, and blood volume. Radionuclide imaging offers a unique way to provide important insight into the pathophysiology of various cardiac diseases by visualizing the sympathetic innervation of the heart. ${ }^{18}{ }^{123} \mathrm{I}-\mathrm{MIBG}$ (metaiodobenzylguanidine), approved by the FDA in 2013, is available and the preferred agent used in clinical practice. The current key semi-quantitative parameters for ${ }^{123}$ I-MIBG cardiac imaging are the heart-to-mediastinum ratio and myocardial washout. ${ }^{19,20}$ These parameters are subject to specific imaging parameters and must be standardized according to the type of imaging device used in order to be clinically useful and accurate. Although probably underutilized in clinical practice, ${ }^{123}$ I-MIBG cardiac imaging shows very great clinical uses and potential. Travin ${ }^{21}$ elegantly summarized the demonstrated and the potential clinical uses of cardiac adrenergic imaging. ${ }^{123}$ I-MIBG cardiac imaging proved to be very useful not only in assessing patients with heart failure and low ejection fraction (and risk stratification in terms of cardiac events) or risk stratification in patients with ventricular arrhythmias associated with heart failure but also in evaluating patients post-cardiac transplant, patients with primary arrhythmic diseases, myocardial ischemia, diabetes mellitus, and the monitoring of chemotherapy toxic effects.

${ }^{123}$ I-MIBG show some minor imaging drawbacks, especially related to the various energy peaks of iodine (with septal penetration of the $529 \mathrm{keV}$ higher energy 
photons) and high liver uptake, both representing some limitations to optimal SPECT imaging. Therefore, different PET radiotracers have been developed for evaluating presynaptic neuronal function such as ${ }^{11} \mathrm{C}$ HED (hydroxyephedrine), ${ }^{18}$ F-LMI 1195 (bromo-fluoropropoxy benzyl guanidine), ${ }^{18}$ F-Fluorometaraminol, ${ }^{11} \mathrm{C}$-phenylephrine, ${ }^{18} \mathrm{~F}$-fluorodopamine, and ${ }^{11} \mathrm{C}$-epinephrine. These agents are mostly used in research projects. Other radiotracers have been developed for assessing postsynaptic neuronal functions and parasympathetic nervous system but their clinical usefulness has not been established yet. Therefore, although not the « ideal » agent, ${ }^{123}$ I-MIBG still remains a very good clinical tool for imaging of cardiac innervation.

\section{INFLAMMATION, INFILTRATIVE DISEASE, ATHEROSCLEROSIS, AND OTHER DISEASES}

Over the last decade, nuclear cardiac imaging has expended significantly with the development of unique and targeted molecular imaging techniques, especially in the evaluation of infiltrative and inflammatory cardiac diseases. It is likely that the use of nuclear cardiologic procedures will significantly expand in the very near future in various fields such as detection of apoptosis, vascular remodeling, angiogenesis, and evaluation of the vascular plaque.

Nuclear cardiology is already actively involved in the evaluation of cardiac amyloidosis with ${ }^{99 \mathrm{~m}} \mathrm{Tc}$ Pyrophosphate, ${ }^{123} \mathrm{I}-\mathrm{MIBG},{ }^{18} \mathrm{~F}-\mathrm{NaF}$, and amyloid binding radiotracers $\left({ }^{99 \mathrm{~m}} \mathrm{Tc}\right.$-aprotinin, ${ }^{123} \mathrm{I}$-serum amyloid $\mathrm{P}$ component or SAP, ${ }^{11} \mathrm{C}$ Pittsburgh $\mathrm{B}$ compound, ${ }^{18} \mathrm{~F}$ florbetapir, ${ }^{18} \mathrm{~F}$-flumetamol, and ${ }^{18} \mathrm{~F}$-florbetaben). The latter three radiotracers have been recently approved by the FDA for beta-amyloid imaging of the brain in patients with Alzheimer's disease but not for cardiac amyloidosis. Their clinical availability will certainly help to prompt evaluation of cardiac infiltration as well.

Myocarditis and sarcoidosis have been studied with various radiotracers such as radiolabeled white blood cells $\left({ }^{99 \mathrm{~m}} \mathrm{Tc}\right.$ or $\left.{ }^{111} \mathrm{In}\right)$, radiolabeled proteins such as $\mathrm{IgG}$ and albumin $\left({ }^{99 \mathrm{~m}} \mathrm{Tc}\right.$ or $\left.{ }^{111} \mathrm{In}\right),{ }^{67}$ Gallium-citrate, and ${ }^{18} \mathrm{~F}$ FDG. The best current radionuclide procedure to investigate myocardial sarcoidosis infiltration is the combined use of perfusion imaging and inflammation imaging with ${ }^{18}$ F-FDG. ${ }^{22,23}$ Different diagnostic combinations may help to monitor response to therapy and assess of the risk in patients with cardiac sarcoidosis. Combination of MPI and ${ }^{18}$ F-FDG findings allows accurate evaluation of cardiac sarcoidosis at different phases of the disease and provides useful information to guide therapy. As with other techniques, large clinical trials are mandatory in order to detail the added value of nuclear cardiology to patient evaluation and outcome. New more specific radionuclides for inflammation detection such as ${ }^{68} \mathrm{Gal}-$ lium-DOTANOC, $\quad{ }^{18} \mathrm{~F}$-fluorothymidine, $\quad{ }^{68} \mathrm{Ga}-$ DOTATOC, and ${ }^{18} \mathrm{~F}$-fluoromisonidazole can certainly offer an interesting alternative to the less specific ${ }^{18} \mathrm{~F}$ FDG.

Anatomic imaging techniques of atherosclerosis have a very limited capacity to identify the hallmarks of the vulnerable plaque. Nuclear cardiology has a unique opportunity to play a pivotal role in the non-invasive assessment of the vulnerable plaque given its ability for molecular imaging. The accumulation of lipids and inflammatory cells within discrete areas of the intima offers potential targets to specifically designed radiotracers. The association of local presence of high macrophage density, lipid-rich necrotic core, neovascularization, micro-calcifications, remodeling, and a thin fibrous cap are the perfect ingredients for the rupture of a high-risk vulnerable atherosclerotic plaque. ${ }^{24}$ Most of the studies for atherosclerosis evaluation are performed with ${ }^{18}$ F-FDG PET/CT for large vessel imaging. Detection of ${ }^{18} \mathrm{~F}-\mathrm{FDG}$ into coronaries is much more technically challenging and limited by interference from normal radiotracer uptake, the quality of the diet preparation, cardiac and respiratory motions (problem for co-registration between PET and CT), and the limited spatial resolution. Therefore, there is a huge clinical need for a more specific and dedicated radiotracer for vulnerable cardiac plaque detection.

Manabe et al. ${ }^{14}$ also discussed the potential use of many other radiotracers, which is beyond the scope of this article. Most of them unfortunately are only in the early stages of animal or human pre-clinical studies, and based on the historical development of radiotracers during the last several decades, only a very few of them will go through all the necessary steps for approval in human studies and ultimately being used in a day-to-day clinical practice. The ideal radiotracer used to study a specific aspect or evaluate cardiologic disease might not necessarily be found in a very near future, given all the numerous characteristics involved and the complexity of the pathophysiology to evaluate as well. However, current and significant advances in imaging devices (including hybrid PET, CT, MR imaging, specially dedicated collimators, and new CZT cameras) and newly improved methods of imaging acquisition and analysis can certainly help to improve the relatively minor «imperfections » of the currently available or potential new radiotracers. Dedicated imaging devices to cardiac imaging, (not only for humans but also for small animal studies such as micro-SPECT and micro-PET cameras coupled to hybrid imaging with $\mathrm{CT}$ or MRI) will be essential in both research and clinical studies. 


\section{CONCLUSION}

In order to advance and thrive, nuclear cardiology needs to go beyond myocardial perfusion imaging which is well established and recognized in the diagnosis and risk stratification of patients with CAD. The ability to image the underlying molecular processes of cardiac diseases is a unique and formidable strength of nuclear cardiology. The quest for the «ideal» radiotracer for myocardial perfusion imaging, cardiac metabolism evaluation, cardiac innervation, inflammation, atherosclerosis, etc., must continue. A close collaboration between radiopharmaceuticals and imaging devices developers and researchers is needed in order to fully take advantage of the unique ability of nuclear cardiology to non-invasively assess all the various aspects of cardiac diseases and to increase its clinical relevance.

\section{Disclosure}

Raymond Taillefer and Francois Harel declare that there is no conflict of interest to disclose.

\section{References}

1. Blumgart HL, Weiss S. Studies on the velocity of blood flow. VII. The pulmonary circulation time in normal resting individuals. J Clin Invest 1927;4:399.

2. Prinzmetal M, Corday E, Spizler RJ. Radiocardiography and its clinical applications. J Am Med Assoc 1949;139:617.

3. Dilsizian V, Taillefer R. Journey in evolution of nuclear cardiology. State-of-the-art paper: will there be another quantum leap with the F-18-labeled myocardial perfusion radiotracers. JACC Cardiovasc Imaging 2012;5:1269-84.

4. Beller GA, Watson DD. Physiological basis of myocardial perfusion imaging with the technetium99m agents. Semin Nucl Med 1991;12:173-81.

5. Dahlberg ST, Leppo JA. Myocardial kinetics of radiolabeled perfusion agents: basis for perfusion imaging. J Nucl Cardiol 1994;1:189-97.

6. Taillefer R, Wackers FJT. Kinetics of conventional and new cardiac radiotracers. In: Iskandrian A, Garcia EV, editors. Nuclear cardiac imaging: principles and applications. 5th ed. New York: Oxford University Press; 2016. p. 58-80.

7. VanTosh A, Garza D, Roberti R, et al. Serial myocardial perfusion imaging with dipyridamole and rubidium- 82 to assess restenosis after angioplasty. J Nucl Med 1995;36:1553-60.

8. Prior JO, Allenbach G, Valenta I, et al. Quantification of myocardial blood flow with Rb-82 positron emission tomography: clinical validation with $\mathrm{O}-15$ water. Eur $\mathrm{J}$ Nucl Mol Imaging 2012;39:1037-47.

9. Higuchi T, Nekolla SG, Huisman MM, et al. A new 18F-labeled myocardial PET tracer: myocardial uptake after permanent and transient coronary occlusion in rats. J Nucl Med 2008;49:1715-22.

10. Maddahi J, Czernin J, Lazewatsky J, et al. Pahe I, first-human study of BMS747158, a novel 18F-labeled tracer for myocardial perfusion PET: dosimetry, biodistribution, safety and imaging characteristics after a single injection at rest. J Nucl Med 2011;52:1490-8.

11. Berman DS, Maddahi J. Tamarappoo BK, et al. Phase II safety and clinical comparison with single-photon emission computed tomography myocardial perfusion imaging for detection of coronary artery disease. J Am Coll Cardiol 2013;61:469-77.

12. Johnson LL, Seldin DW. Clinical experience with technetium-99m teboroxime, a neutral, lipophilic myocardial perfusion imaging agent. Am J Cardiol 1990;66:E63-7.

13. Taillefer R. Technetium-99m teboroxime. In: Taillefer R, Tamaki $\mathrm{N}$, editors. New radiotracers in cardiac imaging: principles and applications. Stanford: Appleton Lange; 1999. p. 49-74.

14. Manabe O, Kikuchi T, Scholte AJHA, et al. Radiopharmaceutical tracers for cardiac imaging. J Nucl Cardiol 2017. https://doi.org/10.1007/s12350-017-1131-5.

15. Otto CA, Brown LE, Scott AM. Radioiodinated branch-chain fatty acids: substrates for beta oxidation. J Nucl Med 1984;25:75-80.

16. Kawamoto M, Tamaki N, Yonekura Y, et al. Significance of myocardial uptake of iodine-123-labeled beta-methyl iodophenyl pentadecanoic acid: comparison with kinetics of carbon-11-labeled palmitate in positron emission tomography. J Nucl Cardiol 1994;1:520-8.

17. Gropler RJ. Imaging myocardial metabolism. In: Iskandrian A, Garcia EV, editors. Nuclear cardiac imaging: principles and applications. 5th ed. New York: Oxford University Press; 2016. p. 518-39.

18. Dae MW, O'connell JW, Botvinick EH, et al. Scintigraphic assessment of regional cardiac adrenergic innervation. Circulation 1989;79:634-44.

19. Travin MI. Cardiac autonomic imaging with SPECT tracers. J Nucl Med 2013;20:128-43.

20. Imamura $\mathrm{Y}$, Ando $\mathrm{H}$, Mitsuoka $\mathrm{W}$, et al. Iodine-123 metaiodobenzylguanidine images reflect intense myocardial adrenergic nervous activity in congestive heart failure independent of underlying cause. J Am Coll Cardiol 1995;26:1594-9.

21. Travin MI. Radionuclide imaging of cardiac innervation. In: Heller GV, Hendel RC, editors. Nuclear cardiology: practical applications. 3rd ed. New York: McGraw Hill; 2018. p. 345-64.

22. Langah R, Spicer K, Gebregziabher M, Gordon L. Effectiveness of prolonged fasting 18F-FDG PET-CT in the detection of cardiac sarcoidosis. J Nucl Cardiol 2009;16:801-10.

23. Blankstein R, OsborneM Naya M, et al. Cardiac positron emission tomography enhances prognostic assessments of patients with suspected cardiac sarcoidosis. J Am Coll Cardiol 2014;63:329-36.

24. Kolodgie FD, Burke AP, Farb A, et al. The thin-cap fibroatheroma: a type of vulnerable plaque: the major precursor lesion to acute coronary syndromes. Curr Opin Cardiol 2001;16:285-92. 